\title{
Annick Fenet, Les dieux olympiens et la mer. Espaces et pratiques cultuelles
}

Préface de Madeleine Jost ("Collection de l'École française de Rome", 509), Roma, École française de Rome, 2016, pp. 709, ISBN

978-2-7283-1065-4, € 60

\section{Corinne Bonnet}

\section{(2) OpenEdition}

Journals

Édition électronique

URL : http://journals.openedition.org/mythos/668

DOI : $10.4000 /$ mythos. 668

ISSN : 2037-7746

Éditeur

Salvatore Sciascia Editore

\section{Édition imprimée}

Date de publication : 1 décembre 2017

Pagination : 168-270

ISSN : $1972-2516$

\section{Référence électronique}

Corinne Bonnet, « Annick Fenet, Les dieux olympiens et la mer. Espaces et pratiques cultuelles », Mythos [En ligne], 11 | 2017, mis en ligne le 24 septembre 2019, consulté le 25 septembre 2020. URL : http:// journals.openedition.org/mythos/668 ; DOI : https://doi.org/10.4000/mythos.668 


\section{Annick Fenet}

Les dieux olympiens et la mer. Espaces et pratiques cultuelles

Préface de Madeleine Jost ("Collection de l'École française de Rome", 509), Roma, École française de Rome, 2016, pp. 709, ISBN 978-2-7283-1065-4, €60.

Corinne Bonnet - Université Toulouse - Jean Jaurès - cbonnet@univ-tlse2.fr

Ce volumineux travail est issu d'une thèse de doctorat soutenue en 1998 par Annick Fenet à l'Université de Paris Nanterre, sous la direction de Pierre Chuvin, et largement, longuement et patiemment remaniée. II est vrai qu'Annick Fenet appartient à ces courageux chercheurs qui, de contrat en contrat, s'impliquent dans des projets collectifs où ils donnent le meilleur d'eux-mêmes, sans nécessairement trouver le temps de cultiver leur propre jardin. Annick Fenet est ainsi devenue, au fil des années, une chercheuse de pointe, auteur de travaux remarquables, sur I'histoire de l'archéologie antique aux XIX et $X X$ siècles. Et pendant ce temps, les Olympiens attendaient... On est donc heureux de voir aboutir la publication de cette thèse sur un sujet important et complexe.

Même si l'ouvrage est imposant, il est important de savoir ce qu'on peut y trouver et ce qui n'y figure pas, de comprendre avec quelle approche, quels questionnements, l'Auteure a abordé son sujet. Elle s'en explique dans l'Introduction (p. 1-14), où elle propose un "Historique de la recherche", puis précise l'" Orientation du sujet ". Dans le prolongement du travail de $\mathrm{D}$. Wachsmuth

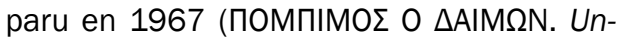
tersuchung zu den antiken Sakralhandlungen bei Seereisen - le mot Sakralhandlungen doit être corrigé dans la bibliographie), il s'agit de s'intéresser avant tout, comme le souligne le sous-titre, aux lieux et aux modalités du culte pratiqué par les gens de mer. Si le titre, quant à lui, insiste sur les dieux et si, toute la première partie du volume leur est consacrée (p. 17-242), A. Fenet est plus à l'aise avec l'inscription des pratiques cultuelles dans l'espace, dans des offrandes et des dédicaces, ce qu'elle appelle les realia (p. 13) qu'avec les configurations de dieux au sein de panthéons, telles que les historiens des religions les étudient. De fait, cela correspond aux compétences spécifiques de l'Auteure, mais suscitent quelques regrets chez les historiens des religions. C'est en tout cas autour de la notion de "culte marin ", définie p. 7 , que le volume est pensé, un culte vécu, pratiqué, qui laisse des traces dans le matériel archéologique et dans les sources écrites. Les questions relatives aux origines de ces cultes marins ne sont donc pas abordées, ce qui semble une sage décision. Faut-il pour autant définir la démarche comme " positiviste " (p. 8-9) par opposition à une approche qui serait " structuraliste " ? Ce genre d'affirmation date sans doute davantage de 1998 que de 2016 ! Là où le positionnement d'A. Fenet aurait mérité d'être précisé et affiné, c'est plutôt dans la relation entre "traditions mythologiques " et "pratiques rituelles ": nous $y$ reviendrons plus avant. En outre, la notion de "dieux olympiens " est-elle vraiment pertinente pour aborder un tel sujet? N'imposet-elle pas un carcan qui finit par laisser de côté des divinités pourtant essentielles pour le sujet, comme Isis ? Car, il est bon de le préciser, l'ouvrage d'A. Fenet couvre un empan chronologique de huit siècles, de l'époque archaïque à l'époque hellénistique (cf. p. 10-11 pour l'explication de ce choix). La catégorie des "Douze Dieux ", comme l'a bien montré S. Georgoudi, est d'ailleurs une construction sujette à des variations importantes ; le Sommaire en mentionne finalement dix (ou onze si l'on compte séparément Déméter et Korè traitées ensemble)! L'exclusion d'Héphaïstos, pour ne prendre qu'un exemple, (cf. p. 242 n. 4 pour ses liens avec le milieu marin) aurait mérité d'être mieux sous-pesée. 
Un des acquis majeurs du travail d'A. Fenet est de montrer que " l'éventail des divinités recevant un culte marin s'avère beaucoup plus large et diversifié que ce que nous apprennent les manuels et l'historiographie traditionnelle " (p. 5). La polyvalence de la puissance divine dont chaque dieu est investi ressort avec force puisque quasiment tous les Olympiens ont maille à partir avec la mer et les gens de mer, avec diverses variations, nuances, spécificités que le travail minutieux d'A. Fenet fait bien émerger. C'est pourquoi ce riche et vaste dossier, bien que "positiviste ", illustre et questionne le fonctionnement complexe du polythéisme (p. 6), un aspect qui n'est qu'effleuré et qui ne bénéficie sans doute pas assez d'une série de travaux récents, dont certains sont bien mentionnés en note, mais qui n'ont pas véritablement contribué à la reformulation des interprétations initiales (celles de la thèse). On songe notamment à la série de publications touchant à la fabrication et à la représentation du divin dans les mondes grec et romain, objet d'un projet collaboratif marquant et fécond, coordonné par Nicole Belayche, entre 2008 et 2011. Ces travaux d'historiens des religions, mais aussi d'archéologues, d'iconologues, etc. auraient sans doute permis d'enrichir les analyses sur la manière dont les prérogatives des dieux en mer, sur le rivage, dans les îles, sur les bateaux ou en faveur des gens de mer, etc. s'articulent, se complètent, créent des collaborations ou des polarités, bref contribuent à la structuration de la " société des dieux ". L'Introduction aurait peut-être pu aussi présenter ce qu'est l'espace maritime : comment les Grecs le définissait-il, le percevait-il, quelle est, comme dirait Fernand Braudel (absent de cette Introduction), la part du milieu dans la relation des Grecs à la mobilité en mer ? en quoi cette mer est-elle corruptrice, comme l'ont montré P. Horden et N. Purcell, " amère " comme disait Platon ? à quel imaginaire est-elle rattachée (cf. M.-C. Beaulieu, The Sea in the Greek Imagination, Philadelphia, University of Pennsylvania Press, 2016, à ajouter dans la bibliographie). S'agissant d'une enquête sur la mer "vécue ", ces éléments de contextualisation des cultes auraient été bienvenus, même si le livre compte déjà plus de 700 pages.

II est structuré en deux parties : tout d'abord "Les divinités olympiennes et la mer " (qui ressemble étrangement au titre du livre !), ensuite "Pratiques cultuelles marines ". La première partie passe en revue les divinités retenues, regroupées en trois chapitres : Athéna et Héra "à la conquête de la mer "; Zeus, Aphrodite, Déméter et Korè pour " la maîtrise de la mer "; Apollon et Poséidon et "la domination de la mer "; Artémis, Dionysos et Hermès pour "les frontières de la mer, ou la mer domestiquée ". II y a évidemment quelque chose d'un peu artificiel dans cette catégorisation, mais elle a le mérite de souligner d'emblée que le rapport à la mer varie et dépend des capacités, compétences et modes opératoires que l'on attribue à chaque divinité, selon les sources, les espaces, les périodes. Un même schéma est appliqué à l'examen de chaque divinité dans sa dimension marine : "les sources d'abord ", littéraires pour commencer, dans l'ordre chronologique, avec des choix, qui ne sont pas toujours suffisamment explicités ; ainsi pour Zeus, passe-t-on en gros d'Homère à Virgile, sans guère d'étapes intermédiaires ; ensuite les " témoignages cultuels ", classés par ordre géographique, en partant de l'Attique, souvent la plus riche en témoignages. Si la documentation archéologique est systématiquement présentée (quoique succinctement), les inscriptions et les monnaies ne sont pas prises en compte de la même manière (p. 12), ce que l'on pourra regretter. L'ampleur du sujet entraîne que le traitement des données reste occasionnellement trop rapide ou superficiel. En revanche, l'ensemble des notices fait émerger un nombre considérable de cultes que d'autres pourront approfondir et analyser en détail : c'est une mine de renseignements qu'A. Fenet met à notre disposition ! Pour revenir à la présentation-type de chaque divinité, sont également examinées les princi- 
pales données iconographiques, avant que tout le dossier soit l'objet d'une analyse synthétique portant sur la géographie du culte et ses modalités, ainsi que sur la " personnalité marine " de la divinité concernée.

Dans la deuxième partie, deux chapitres abordent successivement la présence des dieux sur les bateaux (décor naval et religieux, noms théophores de bateaux, ancres à bord) et les ex-voto à terre (dons de bateaux, ancres, autres offrandes marines). A. Fenet montre bien l'omniprésence des dieux et de leur puissance prophylactique et bienveillante, mais parfois aussi redoutable, à la fois durant la navigation ou la pêche, mais aussi en amont et en aval. Cette seconde partie est d'une richesse impressionnante et justifierait à elle seule la publication si longtemps différée de la thèse. L'ouvrage se termine par une conclusion qui revient sur les dieux, les circonstances et la géographie des cultes. II est en outre pourvu de nombreuses cartes (15), d'un corpus de sources littéraires choisies (" peu aisément consultables ou pour lesquels il n'existe pas à l'heure actuelle de traduction en langue moderne "; p. 533), d'un index thématique des sources littéraires, d'un inventaire des ex-voto navals des inventaires déliens, d'un catalogue des ancres de plomb avec inscriptions théophores et/ou décors, divers tableaux récapitulatifs et une longue bibliographie (p. 616-661), sans oublier divers indices très utiles.

Sur un sujet considérable, aussi vaste que l'immensité de la mer vineuse, A. Fenet apporte des éclairages significatifs au départ d'un matériau riche et diversifié. Nul doute que son ouvrage suscitera bien des prolongements, notamment de la part des historiens des religions qui auront à cœur, notamment, de restituer, dans les divers lieux les plus fréquemment évoqués, Athènes, Délos, Cos, Rhodes, la Sicile, etc., les dynamiques panthéoniques au sein desquelles les prérogatives marines interagissent avec d'autres champs des activités humaines accessibles à la puissance des dieux. 